\title{
A 12 year old girl with acrodysostosis: a rare cause of short stature
}

\author{
Sujeewa Amarasena ${ }^{1}$, M G K. Samanlatha ${ }^{2}$, M H A D de Silva ${ }^{3}$, P Kolombage ${ }^{4}$
}

Sri Lanka Journal of Child Health, 2009; 38: 72-73

(Key Words: acrodysostosis, short stature, exostoses)

Acrodysostosis is a rare disease, first described in 1968 , characterized by short hands and feet with peripheral dysostosis, small nose and mental retardation ${ }^{1}$. Although inherited as an autosomal dominant disorder ${ }^{2}$ most are new mutations. There are just over 50 published cases ${ }^{2}$.

\section{Case story}

A 12 year old girl was brought for investigation of learning difficulty and short stature. Apart from this she had no medical problems. The child had been adopted at one year of age and details about her biological parents were not available. Adopted parents got the baby routinely checked through a medical practitioner prior to adoption and no medical problems were identified. There were no concerns regarding her early development. The child was slow to learn at school. She had short hands and fingers which received the attention of teachers and classmates. Her immunization was complete and diet adequate in calories and proteins.

On examination the height was $131 \mathrm{~cm}(10 \mathrm{~cm}$ below 3rd centile) and weight $25 \mathrm{~kg}$ (4 $\mathrm{kg}$ below $3 \mathrm{rd}$ centile). The OFC was $52.5 \mathrm{~cm}$ (between 25 th and 50th centiles). She had brachycephaly, flat nasal bridge, broad short upturned nose and a flat facies with mild prognathism. Her limbs were short with short, broad hands and feet (Figures 1 and 2).

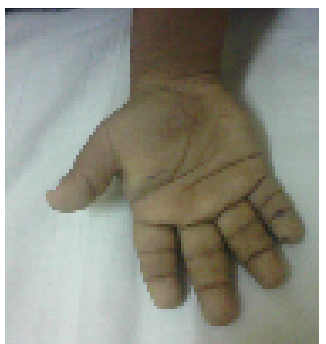

Figure 1: Short, broad hand

${ }_{1}^{1}$ Associate Professor in Paediatrics, Faculty of
Medicine, University of Ruhuna, ${ }^{2}$ Registrar,
University Paediatric Unit, Teaching Hospital,
Karapitiya, ${ }^{3}$ Senior Registrar, University Paediatric
Unit, Teaching Hospital, Karapitiya, ${ }^{4}$ Consultant
Radiologist, Teaching Hospital, Karapitiya.

(Received on 18 September 2008. Accepted after revision on 20 October 2008)

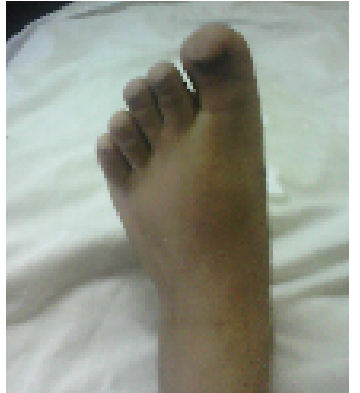

Figure 2 Short, broad foot

The fingers and toes were short. Hair growth was normal. There was no discrepancy in proximal or distal segments of the limbs and both upper and lower segment ratios were normal. There were early signs of breast enlargement compatible with Tanner stage II but other features of puberty were not present. Mental age assessment was around 8 years.

Serum creatinine, blood urea, serum electrolytes, serum calcium, phosphate, alkaline phosphatase and full blood count were normal. Marginal elevation of TSH with normal FT3 and FT4 were found on two occasions. Ultrasound scan of the abdomen did not reveal any abnormality. A skeletal survey showed a bone age of 15 years and exostoses arising from the metadiaphyseal regions of both tibias. There was a mild genu varus deformity and a modelling deformity of bones around the knee joints. Radiographs of the hands and feet showed shortened stubby metacarpals, metatarsals and phalanges. There were inverted "V" shaped depressions (cone shaped epiphysis) in the metatarsals, metacarpals and phalanges bilaterally (Figures 3 and 4).

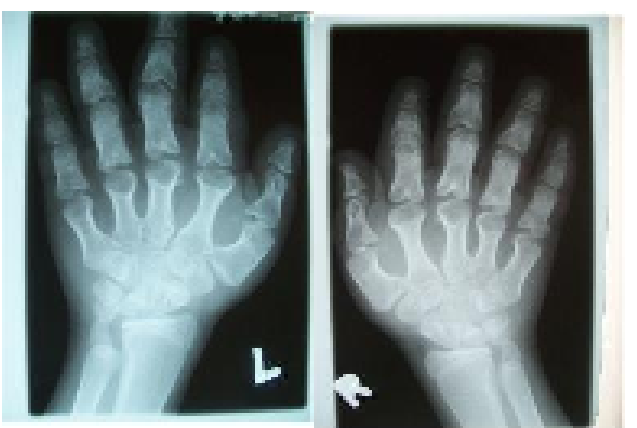

Figure 3 Shortened stubby metacarpals \& phalanges 


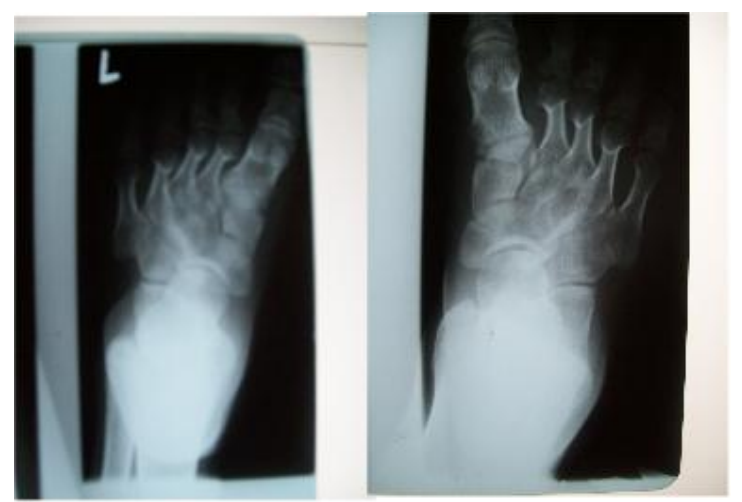

Figure 4 Shortened stubby metatarsals \& phalanges

There were shortened ulna bones. Radiographs of skull, pelvis, chest and the spine were normal. Growth hormone assay showed marginally low GH levels.

\section{Discussion}

This child presented with short stature and learning difficulty. There were dysmorphic features suggestive of a skeletal dysplasia with short limbs. Features of mental retardation, short hands and feet with peripheral dysostosis, brachycephaly, flat nasal bridge, small upturned nose, flat face and mild prognathism are typical of acrodysostosis ${ }^{1,3}$. Cone shaped epiphyses in metatarsals, metacarpals and phalanges were seen. Hypothyroidism is also reported in this condition.

We investigated for possible GH deficiency and hypothyroidism. Some children with certain skeletal dysplasias like achondroplasia have associated GH deficiency and some height is caught up with GH replacement ${ }^{4}$. This child showed marginal elevation of TSH and low GH response for glucagon stimulation tests. However, we did not start the child on thyroxine or $\mathrm{GH}$ replacement.

Most patients with this condition do well except for arthritic complications and progressive restriction of movements of hands, elbows and spine.

\section{References}

1. Jones KL. Smith's recognizable patterns of human malformations. 6th ed. Philadelphia: Elsevier Saunders 2006.

2. Steiner RD, Pagon RA. Autosomal dominant transmission of acrodysostosis. Clinical Dysmorphology 1992; 1:201.

3. Wilson LC, Luttikhuis MEO, Baraitser M, Kingston HM, Trembath RC. Normal Erythrocyte membrane Gs alpha bioactivity in two untreated patients with acrodysostosis. Journal of Medical Genetics 1997, 34 (2); 133-6.

4. Seino Y; Yamanaka Y; Shinohara M; Ikegami S; Koike $\mathrm{M}$ et al. Growth Hormone Therapy in Achondroplasia. Hormone Research 2000, 53 (3); 53-6.

5. Devies SJ, Hughes HE, Familial acrodysostosis: Can it be distinguished from Alb steodystrophy? Clinical Dysmorphology 1992; 1:201. 